\title{
Éléments pour une ethno-sociologie des organisations
}

Gérard Derèze

\section{(2) OpenEdition}

12 Journals

Édition électronique

URL : http://journals.openedition.org/communicationorganisation/1653

DOI : 10.4000/communicationorganisation. 1653

ISSN : $1775-3546$

Éditeur

Presses universitaires de Bordeaux

Édition imprimée

Date de publication : 1 novembre 1993

ISSN : 1168-5549

\section{Référence électronique}

Gérard Derèze, "Éléments pour une ethno-sociologie des organisations ", Communication et organisation [En ligne], 4 | 1993, mis en ligne le 26 mars 2012, consulté le 30 avril 2019. URL : http:// journals.openedition.org/communicationorganisation/1653; DOI : 10.4000/ communicationorganisation. 1653

Ce document a été généré automatiquement le 30 avril 2019.

(c) Presses universitaires de Bordeaux 


\title{
Éléments pour une ethno-sociologie des organisations
}

\author{
Gérard Derèze
}

1 « Explorer les recherches qui analysent les systèmes d'interactions et de représentations à l'œuvre dans le secteur de la communication des organisations »: voilà le thème qui était proposé lors d'une journée d'étude du groupe de contact « Relations publiques et théories de la communication » du FNRS ${ }^{1}$ et qui m'a incité à proposer une réflexion dans ce domaine.

2 J'y ai vu une double opportunité : systématiser quelque peu mon expérience et mes réflexions en matière d'investigation ethnosociologique dans le champ des organisations et soumettre à la discussion (et donc enrichir, critiquer, problématiser davantage) les propositions qui suivent.

3 Le texte qui suit n'est donc rien d'autre qu'une esquisse réflexive qui s'appuie sur des expériences concrètes limitées - dont il ne sera pas rendu compte formellement et explicitement-, qui souhaite dégager quelques éléments de perspective, de programme et de méthodes et qui appelle commentaires, réactions, contestations.

\section{Une approche ethnososiologique : rapides repères}

4 Ces quelques lignes n'ont pour ambition que d'expliciter, très rapidement, ma démarche en indiquant des repères quant à mes sources et références, de délimiter l'approche en en précisant les contours (qui toujours fluctuent, à la différence des frontières) et de montrer mes choix, mes orientations et mes goûts. C'est pourquoi je ne peux et ne veux parler que d'UNE ethnosociologie, celle que je tente de pratiquer, et pas de L'ethnosociologie, sorte de parangon figé et immuable.

5 Un certain nombre d'auteurs utilisent, dans des sens divers et pas toujours clairement définis, le terme d'ethnosociologie. Parmi ceux-ci Guy Barbichon ${ }^{2}$, Daniel Bertaux, Isabelle Bertaux-Wiame, Georges Lapassade ${ }^{3}$. Ce dernier entend par ethnosociologie "une démarche qui transpose à la sociologie le principe de méthode des ethnologues : l'étude 
directe - in situ - de la vie sociale ». Plutôt que de transposition, je préfère, quant à moi, parler d'interfécondation et définir l'ethnosociologie que je tente de pratiquer comme une approche transdisciplinaire et empirique.

6 D'autres auteurs comme Pierre Bouvier qui parle de démarche socio-anthropologique ou Olivier Schwartz qui parle de méthode ethnographique ont largement contribué à la construction de ma réflexion théorique et méthodologique, au même titre d'ailleurs que des ethnologues qui se penchent sur nos sociétés contemporaines et complexes ${ }^{4}$ et des partisans-pratiquants de la sociologie qualitative ou sociologie du quotidien ou encore microsociologie ${ }^{5}$.

7 Resituant également cette approche dans sa filiation interactionniste et qualitative, nous pourrions convenir de définir, provisoirement, approximativement et incomplètement, l'ethnosociologie ${ }^{6}$ comme une approche

8 - situationnelle, c'est-à-dire localisée et contextualisante ${ }^{7}$ ou, comme le préconisait Claude Javeau, tentant de prendre en compte à la fois les situations ponctuelles et les grands systèmes englobants du social

9 - empirique, c'est-à-dire fondée sur l'expérience et le recours indispensable au terrain

10 - dynamique, c'est-à-dire qu'elle doit construire son objet dans le mouvement même de l'enquête ${ }^{8}$ et la spécificité de son approche dans le mouvement même de la recherche

11 - potentiellement distinctive, c'est-à-dire qu'elle peut, au-delà de ce que l'ethnologie a tendance à proposer (des approches totales non parcellaires), tenter de mener des approches segmentaires - qui s'intéressent à des questions spécifiques - en ayant recours, par exemple, à des récits de pratiques focalisés

12 - ordinaire, c'est-à-dire que priorité est donnée au sens commun, au sens donné par les acteurs $^{9}$

13 - cumulative, c'est-à-dire non nécessairement comparative et non superpositionnelle (les réflexions, propositions compréhensives de différentes études ou recherches ne viennent pas se mettre les unes sur les autres mais les unes dans les autres; on pourrait, dans ce sens, oser l'emploi du terme cumulactive)

14 - compréhensive et non explicative ou strictement descriptive, c'est-à-dire qu'elle propose des interprétations localisées

15 - extensive, c'est-à-dire qu'elle doit viser à dépasser l'empirique, le local (plus exactement le localisé) et les interprétations localisées pour tenter de formuler des extensions compréhensives propositionnelles.

16 Plus brièvement encore, on pourrait dire que l'ethnosociologie s'intéresse aux pratiques, aux savoirs, aux interactions et aux représentations ${ }^{10}$ - en les appréhendant comme ordres matériels, symboliques et de communication - ou encore à ce que les gens (les acteurs) font, disent et disent de ce qu'ils font.

17 Je vais tenter, à présent, de montrer que ce type d'approche ethnosociologique est praticable et pertinent dans le champ des organisations et de réfléchir à sa particularité, et donc à ses exigences, contraintes et finalités spécifiques.

\section{Une ethnosociologie des organisations ${ }^{11}$}

«Il y a sans doute quelque laxisme intellectuel à considérer les organisations complexes comme des "cultures", au sens anthropologique du terme. La 
littérature managériale fait ses choux gras depuis quelques années de la «culture d'entreprise ", sans s'être jamais interrogée sur la portée du concept, immensément complexe, qu'elle importait sur ses terres. Mais il y a moyen, à partir notamment de l'ethnographie organisationnelle, de concevoir les organisations comme des microsociétés, avec leurs rites, leurs symboles, leurs codes " secrets et compliqués ", sans tomber dans le pastiche » (Winkin, 1992 :9). et qu'en Europe occidentale son apparition est nettement plus récente. Néanmoins, ces premières recherches européennes d'ethnologie rapprochée " en organisations ${ }^{12}$ permettent de dégager quelques constantes et interrogations. C'est ce que propose Alain Morel (1989:2) lorsqu'il met en évidence trois questions qui ont façonné, selon lui, les grands axes de la recherche ethnologique dans les organisations, et plus particulièrement dans les entreprises :

- Qu'est-ce qu'une culture du travail, considéré du point de vue des savoirs propres à une fabrication ? - axe des savoirs techniques - Dans un cadre local, quelles relations une société entretient-elle avec son ou ses industries ? - axe de l'identité locale -

21 A ce propos, on sera attentif aux propos de Guy Barbichon (1990:182-183) qui insiste sur le fait qu'il faut

« interroger les relations d'accord ou d'opposition qui lient des organisations d'une catégorie donnée à la société environnante par l'intermédiaire, d'une part, des institutions qui embrassent la société nationale dans sa globalité et qui à ce titre sont présentes dans l'enclos organisationnel, et, d'autre part, de la culture, comprise comme l'intériorisation individuelle et collective de manières d'être et de faire dominantes, qui pénètrent naturellement dans le même enclos. » - Comment définir et appréhender ce qu'on appelle la culture d'entreprise?

lors que "simultanément se diffuse une notion de «culture d'entreprise » inspirée par des préoccupations de gestion des relations humaines et s'exprime une demande auprès des ethnologues invités à révéler par l'observation la cohérence propre à une organisation singulière » (Barbichon, 1990 :181), je voudrais tenter de mieux cerner cette notion polysémique de « culture $»^{13}$ en en proposant trois orientations définitionnelles et opératoires qui se combinent aux trois voies majeures de l'investigation ethnosociologique telle qu'elle semble se pratiquer aujourd'hui : l'ethnosociologie dans l'organisation, l'ethnosociologie d'organisation et l'ethnosociologie de l'organisation. Fixant mon attention sur l'objet d'étude, je parlerai donc de culture dans l'organisation, de culture d'organisation et de culture de l'organisation.

Enfin, rappelons qu'essayer d'appréhender et de comprendre les organisations en ethnosociologue c'est justement s'intéresser à la culture comprise comme ordres matériels et techniques (y compris les savoir-faire et les compétences), symboliques et de communication ${ }^{14}$ » et, donc, relier les trois questions synthétiques et programmatiques proposées par Alain Morel.

Tentons maintenant de distinguer ces trois voies majeures.

\section{Ethnosociologie dans l'organisation}

Aujourd'hui un certain nombre d'observations (rôle des services de communication, des attachés de presse, multiplication des agences en tout genre...) renforcent l'affirmation de 
Claude Riveline (1983 : 39) selon laquelle les organisations sont « des entités opaques au regard étranger. Elles ne livrent spontanément que des apparences soigneusement préparées, telles que leurs façades, leurs catalogues et leurs rapports annuels d'activité. » Il est donc indispensable de pénétrer ces lieux de façon approfondie si l'on veut « rendre intelligibles ces univers culturels singuliers » (Morel, 1989:1) qui participent (produisant et étant produits) à l'univers culturel plus global.

Pour certains auteurs, «l'entreprise ${ }^{15}$ a toutes les caractéristiques d'une micro-société et, en particulier, elle est le siège de phénomènes culturels importants» (Le Boeuf, Mucchielli, 1989:29) et donc en l'étudiant, on peut arriver à dire quelque chose de global sur la société.

« Le monde du travail et de la production propose, derrière ses invariants, un vaste champ où, sous les concepts d'entreprise, de corporation, de métier, de profession, de secteurs ou de sites industriels, et à leurs croisements, se composent, préexistent ou se reproduisent des entités que l'étude peut identifier et analyser. C'est en s'attachant à celles dont le travail est le principe, que l'on peut contribuer à un certain renouvellement de la compréhension des faits sociaux contemporains. » (Bouvier P., 1989: 20)

L'ethnosociologie dans l'organisation peut alors prendre trois directions principales :

1. l'organisation est considérée comme un microcosme, une réduction de la société globale $^{16 / 17}$.

30 2. l'organisation est étudiée dans le cadre d'une approche plus large et englobante : par exemple, un atelier de fabrications métalliques en difficulté dans le cadre de l'étude d'une sous-région en voie de désertification industrielle ou un centre de services intégrés à destination des populations immigrées dans le cadre de l'étude de la banlieue d'une grande ville,

31 3. l'organisation est étudiée dans le cadre d'une approche segmentaire qui la prend (aussi) comme terrain d'investigation : à titre d'exemple, les usages des postes téléphoniques à mémoire, l'apparition dans les conversations des sujets sportifs traités par les médias, les rapports intergénérationnels,...

L'organisation est alors le terrain et non l'objet véritable, central de la recherche, ce qui est finalement visé la dépasse toujours: la société, une région ou une problématique particulières.

\section{Ethnosociologie d'organisation}

33 On peut constater que bon nombre des recherches qui se sont déployées avaient pour point de départ un problème ou des difficultés. Les ethnologues ont été, dès lors, amenés à investir l'organisation à la demande de ses responsables afin de proposer des solutions. C'est ainsi qu'on a pu voir se développer des interventions-conseils ${ }^{18}$ à côté des plus habituelles recherches critiques, comparatives ou exploratoires.

Ajoutons que "parmi les voies privilégiées empruntées par les autorités de certaines organisations pour permettre la réalisation de leur projet d'entreprise, il y a l'adoption d'une stratégie globale de communication qui vise certains publics internes et externes, assure la promotion d'un ensemble de valeurs-repères caractérisant la culture " officielle » de l'organisation et marque la dynamique de l'organisation de même que ses multiples rapports avec divers segments de l'environnement » (Beauchamp, 1992 : 153). 

fonctionnement et de rapports (aux autres, à l'organisation,...) de normes, valeurs, symboles qui réunissent les membres autour d'une finalité commune. Reprenant les idées de Deal et Kennedy, M. Beauchamp met l'accent, dans cette perspective, sur les valeurs qui « fournissent une sorte de direction commune à tous les membres de l'organisation » (1992: 154). En reprenant les termes de Gamst ${ }^{19}$ (1984: 12), on peut dire que les conseillers vendent avec profit une culture d'organisation ${ }^{20}$ comme l'on vend une spécialité pharmaceutique.... recettes de cuisine visant à obtenir l'adhésion des travailleurs aux valeurs de la direction. La culture d'organisation ou d'entreprise devient alors « un facteur de rationalisation des entreprises, non plus seulement par la technique et par la force hiérarchique, mais aussi par le culturel et le consensuel » (Chaskiel, $1991: 5)$.

La culture d'entreprise sert «pour mobiliser l'ensemble du personnel sur une identité unique à laquelle chacun peut adhérer » (Caune, 1991 : 115) et est, alors, envisagée comme «la variable sur laquelle agir pour provoquer les transformations économiques et techniques jugées nécessaires " (Chaskiel, 1991: 11) ${ }^{21}$. Ceci m'apparaît pour le moins restrictif et normatifi ${ }^{22} \mathrm{du}$ fait, notamment, de l'instrumentalisation outrancière et de la tendance volontariste et managériale à l'homogénéisation. Mettant en garde contre ces abus, « certains auteurs, comme Philippe d'Iribarne ${ }^{23}$, ont souligné le risque qu'il y avait à gérer une entreprise à travers une approche « monolithique, cohérente et immuable » de la « culture d'entreprise ». Pour cet ingénieur en chef des mines, directeur de recherches au CNRS, une « culture d'entreprise reprend, articule et complète de manière propre un ensemble de matériaux provenant aussi bien de cultures nationales, que de cultures régionales, professionnelles, de groupes sociaux ${ }^{24}$, etc »(Bonnafous, 1992: 172). Cependant là où ces stratégies, techniques, tactiques - allant de la stimulation au contrôle de conformité culturelle - sont prônées et utilisées, elles deviennent aussi, pour l'ethnosociologue, objet d'étude.

Les cercles de qualité, de créativité, le service communication et ce qu'il met en place,... deviennent alors objets de recherche.

« Le concept de culture d'entreprise ${ }^{25}$ nous paraît utile lorsqu'il peut rendre compte de la dynamique des différents éléments qui la constituent, à un moment donné, et décrire un certain état des forces antagonistes, ou parallèles, ou momentanément convergentes, qui proposent des structurations différentes du social.

Sauf à prendre pour vérité la parole (ou architecture) de tel groupe social et à considérer que la culture d'entreprise qu'il promeut, impose ou négocie est la culture de l'entreprise, on ne peut pas appréhender cette culture en terme de substance, d'immanence, ou de trésor à sauvegarder : c'est comme enjeu et résultat d'un processus à analyser qu'elle devient intelligible et prend valeur explicative » (Mairot, 1989 : 239).

De mon point de vue, on pourrait placer les «recherches-actions» dans cette même perspective de la culture d'organisation (même si cela peut paraitre paradoxal). Certes, elles ont très souvent des finalités politiques et des a priori différents, voire opposés à ceux des dirigeants, mais elles reposent aussi sur cette dynamique nécessairement volontariste qui fait qu'elles doivent se construire, s'évaluer et se comprendre de façon auto-centrées, c'est-à-dire avant tout (voire uniquement) par rapport à elles-mêmes. 


\section{Ethnosociologie de l'organisation} avec les recherches anthropologiques classiques peuvent s'affirmer riches, intéressants mais dangereux. En effet il faut être conscient que les transferts conceptuels ont toujours quelque chose de périlleux et peuvent nous faire "tomber dans le pastiche", comme l'écrivait Y. Winkin ou nous faire basculer dans l'analogie factice, réductrice et improductive si nous ne nous montrons pas vigilants.

«Des phénomènes de ritualisation, autre emprunt à l'anthropologie, instituent et préservent les identités professionnelles et sociales. Les conditions socioprofessionnelles produisent d'abord de l'obligation. L'exécutant s'efforce de concilier les données techniques et organisationnelles avec ses attentes éthiques. Il met en place, individuellement et collectivement, un certain nombre de conduites aptes à tempérer, réinterpréter voire nier la réalité à laquelle il est assigné. Le bloc sociotechnologique ${ }^{26}$ dispose, dans ses professionnalités et dans ses sociabilités, de façons de faire et de façons de penser liées aux tâches et aux fonctions. " (Bouvier P., 1989 : 21)

\section{Distinctives}

Il est possible de travailler, dans cette perspective, sur tel service, tel réseau, ou telle sous-population particulièrere, puisque dans toute grande organisation, «il existe des cultures socio-professionnelles spécifiques" (Ribeill, 1984: 251). De même, "des observations ethnographiques peuvent très bien concerner, non ces fonctions particulières, finance, marketing, gestion de la production, ressources humaines,...) de l'entreprise mais leurs interfaces » (Matheu, $1983: 44$ ).

Il peut y avoir aussi un grand intérêt à travailler sur des problématiques particulières. Nous partageons, dans cette direction de recherche, l'avis d'André-Georges Haudricourt ${ }^{27}$ qui avance que "n'importe quel objet, si vous l'étudiez correctement, toute la société vient avec ». On peut ainsi mener des observations et des recherches approfondies, entre autres, sur la production langagière, par exemple les journaux d'entreprise, les notes, feuilles d'information syndicale,... «c'est-à-dire toute la production de sens sur la cohésion du groupe ou sur les valeurs de celle-ci, qui est donnée à l'occasion de discours et d'écrits sur l'entreprise» (Pierzo, 1985: 152). Une analyse rigoureuse ${ }^{28}$ de ces productions formelles, ainsi que de leurs situations de production, de diffusion et de consommation peut faire surgir des éléments pertinents de compréhension. 
44 Lors d'études menées dans des entreprises et des organisations sociales ou culturelles, nous avons travaillé, entre autres, sur les usages rédactionnels des termes de légitimation de l'action. Nous avons ainsi pu dégager des «topos $»^{29}$ tels que, à titre d'exemple : «se donner à fond ", "être en première ligne ", «mouiller sa chemise » ou encore "rester proche de la base ».

45 Dans la discussion qui suivait l'intervention de Pierzo (1985), Isaac Joseph proposait, lui, de travailler sur ce qui se dit sous forme de conversation, de bavardages, de ragots, de rumeurs, en d'autres mots sur les productions informelles.

46 En m'inspirant, entre autres, de Denis Guigo et de mon expérience personnelle, je me propose de relever quelques axes de recherche et d'investigation qui peuvent se révéler productifs et intéressants pour élaborer des interprétations localisées et des extensions compréhensives propositionnelles ${ }^{30}$ :

47 - les constitutions de coalitions d'individus ainsi que les circonstances et modalités de leur mobilisation (chaque personne appartenant à plusieurs coalitions virtuelles différentes qui ne se trouvent pas activées, actualisées en même temps, il est indispensable de s'interroger sur les situations et les conditions de mobilisation)

48 - l'usage du vouvoiement, du tutoiement ou l'utilisation du prénom en présence ou en l'absence des personnes qui peuvent jouer un rôle de valve dans la circulation de l'information.

49 - le temps de l'organisation

«Ce niveau d'analyse se réfère au temps généré par diverses organisations dont le fonctionnement, les horaires, l'étalement des activités ont pour propriété de structurer le temps d'une manière qui peut leur être spécifique. De tels temps interfèrent avec d'autres niveaux; ainsi le fonctionnement d'une entreprise de transformation, selon le système des trois-huit ou travail posté, influence les temps personnels des ouvriers tout en étant inscrit dans le temps macrosocial du travail à l'échelle de la ville ou de la société » (Pronovost, 1986 : 11)

50 - Les modes d'utilisation (et de contournement) des circuits officiels, explicites et des circuits officieux (cachés), implicites de circulation de l'information

51 - l'apparence vestimentaire

52 - les processus de figuration ${ }^{31}$

53 - le cadre de vie (mobilier, gestion de l'espace, décoration, répartition du personnel dans les locaux, accueil,...)

54 - les façons de se nourrir : comment mange-t-on ? qui mange quoi, où, quand, avec qui ?

55 - les coulisses, la scène ${ }^{32}$, les secrets et les pseudo secrets qui peuvent faire fonctionner (volontairement ou non) la machine à rumeurs.

56 - les rites qui sont « des catalyseurs d'information qui manient essentiellement le temps et le lien social » (Charasse, 1989 : 228)

57 - les figures emblématiques et référentielles, les événements fondateurs... tels qu'ils apparaissent dans les actes officiels (discours, publications), dans les conversations et dans les relations avec l'extérieur où ils peuvent opérer comme déclencheurs, mots de passe ou signe de reconnaissance (d'appartenance)

58 - la mobilité spatiale (dans et hors l'organisation) et institutionnelle,...

59 Une foule d'autres pistes de travail, de recherche, de réflexion peuvent permettre de proposer des interprétations localisées et - par contextualisation, accumulation et 
ouverture - des extensions compréhensives propositionnelles. Il s'agit cependant de rester prudent et d'interroger nos propositions compréhensives et théoriques sur leur « inévitable contrepartie d'ignorance » (Laurens, 1991 : 93). J. Caune a bien vu, me semble$\mathrm{t}$-il, un des risques qui menace notre travail : « la culture d'entreprise (est) constituée de signes. Pourtant, à mettre sur le même plan les logos, les images visuelles, les habitudes vestimentaires, les comportements obligés (tutoiement, chemisette blanche à manches courtes,...) la culture des signes ne risque-t-elle pas de verser dans l'illusion sémantique ? Alors que la culture est d'abord production de pratiques » (1991:117).

\section{Culture de l'organisation}

Pour aborder la culture de l'organisation, « les habitudes de travail, les règles et normes informelles, les images collectives, les manières de percevoir et de comprendre... ( Le Bœuf, Mucchielli, 1989 : 29) deviennent orientations de recherche capitales.

61 Et dans une acception véritablement ethnosociologique, la culture de l'organisation «se réfère à une notion extensive : spécificité culturelle de l'entreprise inscrite dans un corps social et culturel donné » (Barbichon, $1990: 183)$. Elle est « combinaison dynamique (...), assemblage qui fait la spécificité ultime de telle usine, qui caractérise en dernière analyse la façon dont on est forgeron dans cette entreprise, routier dans telle autre » (Mairot, $1989: 241)$

\section{Trois réflexions pour conclure}

Peut-être, certains lecteurs auront-ils perçu de l'agacement, voire de l'intransigeance dans mes propos? C'est possible. En effet, je supporte assez mal la confusion volontaire et trompeuse entretenue par certains pseudochercheurs ou pseudo-scientifiques dans le champ professionnel de l'intervention (conseil, consultance,...). Cette confusion repose, parfois ou souvent, c'est selon, sur l'illusion, le détournement et «l'appauvrissement qu'entraîne une simplification trop sommaire " - comme disait Gide -. Cela me parait inacceptable à de nombreux points de vue : déontologique, éthique, heuristique.

63 Je ne cherche évidemment pas à baliser ou à contraindre des pratiques professionnelles autres que celles qui s'inscrivent dans une perspective ethnosociologique ${ }^{33}$. N'étant ni responsable d'organisation, ni expert-conseil ${ }^{34}$, je ne peux donc parler que de la recherche et de l'investigation, telles que je tente de les pratiquer.

\section{Exercice de la recherche}

64 L'ethnosociologue qui travaille dans des organisations ou entreprises doit se faire connaître et reconnaître par les membres pour diverses raisons : clarté et déontologie, accessibilité à des secteurs et segments de l'entreprise non accessibles à un travailleur de tel ou tel service - qui lui aussi est marqué au sein de l'organisation -.

«Nous pensons qu'un poste non directement connoté à la production peut instaurer cette distanciation que postulent certains anthropologues, et ainsi être à même de mieux répondre à des exigences de déontologie minimale entre observateur et observé, tout en facilitant la lisibilité des uns et des autres par un balayage large de l'ensemble du champ» (Bouvier, 1989 : 41-42). 
65

If fadra aussi tenter, dans le processus de la recherche, de percevoir les difficultés et les perturbations qu'engendre notre présence. Dans ce sens, Nathalie Heinich, parlant de l'«observation participante» et reprenant un passage de Goffman (1991), note que « même lorsque la population étudiée est prévenue, elle ne sait pas exactement quels sont les faits qui intéressent le plus le sociologue, et par conséquent quelles apparences risquent d'être discréditées » (1991: 942). Il est évident que la perception que les membres, les informateurs, les gens vont avoir, ainsi que les réactions à cette perception et à la personne de l'ethnosociologue vont donner une coloration singulière aux situations de co-présence et, parfois, alimenter des conversations et, donc, nourrir des représentations. l'ethnosociologue ne se trouve pas coupé de l'ensemble de la population qu'il veut étudier par le fait d'être emprisonné dans ses relations avec un sous-groupe particulier qui se l'approprie, voire l'utilise.

\section{Chacun son rôle ${ }^{35}$}

67 Lorsqu'ils co-existent, il m'apparaît qu'il y a de sérieux problèmes de compatibilité entre les rôles d'ethnosociologue - qui essaie de comprendre - et de conseiller - qui vérifie ou stimule l'ajustement à la norme ${ }^{36}$-. A mon sens, ils ne peuvent être cumulés, c'est-à-dire exercés dans le même temps dans le même champ d'investigation. Cependant libre à chacun - en fonction des circonstances, des possibilités et contraintes et de ses choix éthiques, déontologiques et professionnels - de devenir conseil(ler) après avoir été ethnosociologue. Mais, même dans ce rôle de conseil(ler), qu'il exercerait par après ou par ailleurs, l'ethnosociologue devra avertir les commanditaires et les différents publics qu'il n'y a jamais de solution automatique, garantie et unique ${ }^{37}$ car le contexte et la situation et, donc, la problématique varient toujours.

Ceci n'empêche pas, d'autre part, les conseil(ler)s de métier de s'inspirer de méthodes ethnologiques. Cependant les risques sont bien réels : par exemple, en fonctionnant - et donc en vendant - sur l'illusion conceptuelle, plutôt qu'en s'interrogeant sur la portée heuristique, épistémologique et pratique des transferts conceptuels - ce qui est une absolue nécessité pour l'ethnosociologue. A propos de l'illusion, C. Le Moenne (1991: 165) note que «certains bureaux d'études déclarent ne pas vouloir intervenir dans la mise en œuvre de leurs recommandations parce qu'il n'est pas possible d'être à la fois juge et partie ». A première vue, cette position peut sembler proche de celle de l'ethnosociologue mais l'auteur ajoute que :

« cette affirmation qui vise à accréditer auprès du client potentiel le sentiment d'une approche distanciée est évidemment un pur argument publicitaire, mais qui manifeste clairement l'empiricité sous-jacente: la prétendue objectivité est ici fondée sur l'illusion d'une possible neutralité épistémologique qui permettrait de recueillir le fait brut, dans sa totale virginité, pour le restituer comme réponse vraie aux questions du client. Accessoirement, cette position permet une distanciation, non vis-à-vis des faits, mais vis-à-vis des éventuelles récriminations d'un client livré à lui-même au moment de la mise en œuvre des recommandations, et auquel on pourra, le cas échéant, conseiller... un cabinet ami » (Le Moenne, 1991,166).

Malgré ces risques de détournement et de manipulation stratégique ${ }^{38}$, il n'y a pas lieu de revendiquer un quelconque monopole d'usage ou d'inspiration en matière ethnosociologique. 
70 Il est donc très clair que, pour moi, l'ethnosociologie ne peut être ni incantatoire ni prescriptive. Etant compréhensive, elle ne peut placer l'ethnosociologue en situation de prendre les décisions. Elle est radicalement différente de la littérature qui veut donner au décideur les clés du succès ainsi que de la logique d'intervention de la plupart des cabinets-conseils et autres agences de communication. De ce point de vue, je rejoins la position d'Yves Winkin (1992:10) quand il écrit :

les organisations «ne peuvent confier l'ensemble de leurs activités de communication à des cabinets indépendants. Ceux-ci peuvent réaliser des actes techniques précis mais les opérations fondamentales doivent être prises en charge par des agents intégrés de longue date dans l'organisation. Si on veut être cohérent avec une vision orchestrale ${ }^{39}$, il ne peut d'ailleurs en être autrement: il faut pleinement être membre de la culture pour pouvoir l'exprimer. Par contre, il faut parfois un observateur extérieur à cette culture pour en saisir les traits. C'est à l'anthropologue de reconstituer les éléments oubliés de la "partition » et de les rendre à ses utilisateurs ».

\section{Qui paie ${ }^{40} ?$}

71 Instances extérieures, patrons, syndicats,... De toute façon, il faut à chaque fois négocier les conditions de travail ${ }^{41}$, de reconnaissance (par l'ensemble des membres), les conditions des retours d'information permettant la réappropriation, les conditions de divulgation de l'analyse. Si on se limite à la publication d'un rapport final destiné aux seuls commanditaires, il faut, dès le départ, en informer les informateurs.

Le retour d'information peut poser problème parce qu'essayer de comprendre, c'est aussi déconstruire et il n'est pas sûr que l'on peut, dans une organisation, vivre tout ce que l'on comprend ni comprendre tout ce qu'on vit. Il en va d'ailleurs de même pour l'ethnosociologue.

73 Enfin il faut être conscient que les perturbations que notre présence va occasionner (ainsi que les dangers et les risques qui y sont liés ${ }^{42}$ ) ne seront acceptées que si, en retour, les commanditaires internes à l'organisation et ceux qui nous accueillent y trouvent un bénéfice, fut-il symbolique. Ceci pose, d'une autre manière la question des rapports entre recherche fondamentale (Gamst parle de recherche de base) et recherche appliquée.

74 Conscient d'être resté, dans ce qui vient d'être avancé, imprécis, schématique et brouillon (mais peut-il en être autrement à ce stade de la réflexion?), je souhaite, pour conclure provisoirement, reprendre et faire miennes deux interrogations de $\mathrm{C}$. Le Moenne qui me paraissent cruciales dans la perspective du développement de l'approche ethnosociologique des organisations :

"Que dire de l'absence quasi totale d'études indépendantes au sens "d'indépendantes des problématiques managériales» voire au sens de "non commercialement intéressées", sur l'état réel des communications - et donc de la vie ${ }^{43}$ des entreprises?

Que dire de la domination sans partage de l'idéologie du professionnel jusque dans l'Université, qui favorise la réception non critique des discours enchantés trop souvent mystificateurs » $(1991: 174)$ 


\section{BIBLIOGRAPHIE}

\section{RÉFÉRENCES}

Althabe G., « Introduction », Sociétés industrielles et urbaines contemporaines, Paris, Maison des Sciences de l'Homme,1985 pp. 1-12.

Auge M., Non-lieux. Introduction à une anthropologie de la surmodernité, 1992, Paris, Seuil.

Barbichon G., « L'ethnologie des organisations », Ethnologie française, 1990 vol. XX, n² 2, pp. 177-188.

Bautier R., Nicolas Y., « Les zéros du management », Les cahiers du LERASS, 1991 n² 23, pp. 19-32. Beauchamp M., Projet d'entreprise et stratégie globale de communication », Les nouveaux espaces de l'information et de la communication, Lille, $19928^{\mathrm{e}}$ congrès SFSIC, pp. 151-157.

Bonnapous S., " Communication interne et identité universitaire ». Les nouveaux espaces de l'information et de la communication, Lille, $19928^{\mathrm{e}}$ congrès SFSIC, pp. 167-174.

Bouvier P., Le travail au quotidien, 1989, Paris, PUF.

Breton P., Entre le monde de l'informatique et la « culture de la communication », L'ordre communicationnel, Paris, La documentation française et CNET-ENST, 1989, pp. 65-74.

Caune J., Identité et culture : concepts fondateurs ou phénomènes de mode. Les cahiers du LERASS $n^{\circ} 23$, 1991, pp. 105-119.

Charrasse D., Rites corporatifs et stratégies d'entreprises dans le bassin sidérurgique de Longwy, Cultures du travail, Paris, Maison des Sciences de l'Homme, 1989, pp. 221-234.

Chaskiel P., Mode des concepts, mode sans concepts, Les cahiers du LERASS, 1991, n² 23, pp. 5-18.

Dereze G., Eléments pour une ethnosociologie des objets domestico-médiatiques, Recherches sociologiques, Louvain-la-Neuve, 1990, vol. XXI, n 3, pp. 307-321.

Durand J-P., « L'entreprise comutante », L'ordre communicationnel, Paris, La documentation française et CNET-ENST, 1989, pp. 133-146.

« Ethnographie des organisations », numéro spécial, Formation et gestion, printemps 1986.

Floris B., "Un espace public pour l'entreprise », Les nouveaux espaces de l'information et de la communication, Lille, 1992, $8^{\mathrm{e}}$ congrès SFSIC, pp. 415-421.

Gamst F., L'ethnologie industrielle nord-américaine, Sociétés, 1984, vol. 1, nº 2, pp. 11-12.

Goffman, Les rites d'interaction, Paris, 1984, Minuit.

Goffman, Les cadres de l'expérience, Paris, 1991, Minuit.

Gosselin G., Contribution à une anthropologie du travail, Cahiers internationaux de sociologie, 1966, vol. XLI, pp. 133-150.

Guigo D., Perspectives ethnologiques dans les organisations modernes, L'Homme, 1992, vol. XXXII, $\mathrm{n}$

- 121, pp. 47-65. 
Heinich N., Pour introduire à la cadre-analyse Critique, 1991, n5 535, pp. 936-953.

Iribarne (d') P., La logique de l'honneur. Gestion des entreprises et traditions nationales, Paris, 1989, Seuil.

Joseph I., « Urbanité et ethnicité », Sociétés industrielles et urbaines contemporaines, Paris, Maison des Sciences de l'Homme, 1985, pp. 135-146.

Laurens P., La fureur de dire. Culture d'entreprise et communication, Les cahiers du Lerass, 1991, $\mathrm{n}^{\circ} 23$, pp. 93-104.

Le Boeuf C., Mucchielli A., 1989. Le projet d'entreprise, Paris, PUF.

Le Moenne C, Le rôle des consultants en communication, Les cahiers du LERASS, 1991, n 23, pp. 157-174.

Mairot P., Les usines-pensionnats au XIX ${ }^{\mathrm{e}}$ siècle dans le Dauphiné: culture et religion d'entreprise, Cultures du travail, Paris, Maison des Sciences de l'Homme, 1989, pp. 235-249.

Mathieu M., Ethnographie et sciences de la gestion, Enseignement et gestion, 1983, numéro de printemps, pp. 43-49.

Molitor M., «L'herméneutique collective », in Remy J., Ruquoy D. (eds), Méthodes d'analyse de contenu et sociologie, Bruxelles, Facultés universitaires Saint-Louis, 1990, pp. 19-35.

Monod E., La nouvelle information et communication d'entreprise et la crise du paradigme idéologique moderne, L'ordre communicationnel, Paris, La documentation française et CNET-ENST, 1989, pp. 101-132.

Morel A., «Introduction », Cultures du travail, Paris, Maison des Sciences de l'Homme, 1989, pp. 1-15.

Petonnet G, Pouchelle M-C, Le rôle de l'ethnologue dans sa société, L'autre et le semblable, Paris, Presses du CNRS, 1989, pp. 183-191.

Pialoux C, Chroniques Peugeot, Actes de la recherche en sciences sociales, 1984, n 54, pp. 57-69.

Pierzo D., Culture interne et lien social dans la société Calor, Sociétés industrielles et urbaines contemporaines, Paris, Maison des Sciences de l'Homme, 1985, pp. 147-154.

Productions symboliques ouvrières. Ethnologie française, tome 14, $\mathrm{n}^{\circ} 2,1984$.

Pronovost G., Le temps dans une perspective sociologique et historique, Revue internationale des sciences sociales, $\mathrm{n}^{\circ}$ 107,1986, pp. 5-19.

Ribeill G., Culture d'entreprises : le cas des cheminots des compagnies à la SNCF, Cultures du travail, Paris, Maison des Sciences de l'Hhomme, 1989, pp. 251-265.

Riveline C, Pour une ethnographie des organisations, Enseignement et gestion, $\mathrm{n}^{\circ}$ de printemps, 1983 , pp. 39-43.

Sainsaulieu R., Segrestin D., Vers une théorie sociologique de l'entreprise, Sociologie du travail, vol. XXVIII, 1986, n 3, pp. 335-352.

Schlanger N., Le fait technique total. Terrain, 1991, n 16, pp. 114-130.

Villette M., Une technologie sociale d'ingénieur-conseil. Actes de la recherche en sciences sociales, $\mathrm{n}$ - 54, 1984, pp. 45-56.

Winkin Y., Communication et relations publiques: quelles théories pour quelles pratiques, in MANGELINCKX B. (ed), Communication et relations publiques, 1992, Bruxelles, De Boeck. 


\section{NOTES}

1. Le présent article s'inspire largement du contenu de mon intervention à cette journée du groupe de contact FNRS - Fonds National de la Recherche Scientifique-, Louvain-la-Neuve, le 18 mai 1992.

2. Dans un article qui concerne notre propos, G. Barbichon (1990) parle, sans distinction d'ethnosociologie, de socio-ethnologie et de socio-ethnologie.

3. La passade G. L'ethnosociologie. Paris, Méridiens Klinckieck, 1991. Dans cet ouvrage, l'auteur montre en quoi l'école de Chicago et l'etho-méthodologie ont nourri et inspiré son approche ethnososociologique.

4. Dont Marc Augé, Gérard Althabe, Georges Balandier,... et plus globalement le courant qu'on a coutume d'appeler l'ethnologie de la France.

5. Je pense particulièrement a des auteurs comme Christian Lalive d'Epinay, Claude Javeau, Michel de Certeau ou encore Edgar Morin et sa sociologie du présent.

6. J'écris ethnosociologie en un seul mot afin de mieux marquer l'association et l'interpénétration des contenus théoriques disciplinaires. Pour une bibliographie plus complète, on pourra se référer a «La croisée des regards. Eléments pour une ethnosociologie active » (G. Derèze, a paraître).

7. Ce que noua étudions est en étroite interaction avec l'environnement, des systèmes plus larges ou englobants. C'est pourquoi il est indispensable de veiller a inscrire la recherche localisée dans des perspective " plus larges et des ouvertures multiples. A titre d'exemple, on se référera a d'autres études (y compris quantitatives, historiques), on tentera de mener des observations " plus larges, on élargira les procédures de recherche en rassemblant et analysant, si cela est praticable et pertinent, un corpus de vignettes descriptives. Par vignettes descriptives, il faut entendre les coupures de presse, les extraits de romana, de bandas dessinées ou de films, les images publicitaires,... tous ces éléments qui sont utiles «pour mettre au four les caractéristiques d'un segment d'expérience (...) On a là quelque chose de facilement disponible, une sorte de fonds commina de l'expérience familière » (Goffman. 1991 :24).

8. Comme le propos Yves Delaporte, «L'objet et la méthode. Quelques réflexions autour d'une enquête d'ethnologie urbaine ", L'Homme. n 97-98, p.168.

9. Ce point de vue rejoint, en quelque sorte, la perspective ethnométhodologique (le langage ordinaire dit la réalité sociale, la décrit et la constitue dans le même temps) et interactionniste «L'authentique connaissance sociologique nous est livrée dans l'expérience immédiate, dans les interactions de tous les jours. Il faut d'abord prendre en compte le point de vue des acteurs, quel que soit l'objet d'étude, puisque c'est à travers le sens qu'ils assignent aux objets, aux gens, aux symboles qui les entourent, que les acteurs fabriquent leur monde social» (Coulon A. L'ethnométhodologie. Paris, PUF, 1987, p. 11.

10. Voir Derèze, 1990.

11. Afin d'éviter les malentendus au moment où nous entrons dans le vif de la réflexion, je propose d'entendre par "organisation ", tout groupement permanent de personnes ayant pour but la production de biens matériels ou de services et astreint à une condition plus ou moins stricte d'efficacité » (SCHEID J-C Les grande auteurs en organisation. Paris, Dunod. 1990. p. 1 ou. m'inspirant d'une définition de l'entreprise que donnent Claude Le Boeuf et Alex Mucchielli (1989:5). toute collectivité d'hommes et/ou de femmes qui (se) sont rassemblés pour atteindre un objectif particulier et déterminé, marchand ou non marchand- qui dépasse les capacités Individuelles de chacun. En d'autres mots, nous parlerons des entreprises, des collectivités locales, des institutions publiques ou privées et des associations. 
12. «Ethnologie rapprochée » qui n'est en aucun cas, pour reprendre l'expression de Marc Augé (1992 : 18) - qui soulignait que les raisons qui avaient amené les ethnologues à travailler sur nos sociétés occidentales étaient, avant tout, des raisons positives - « une anthropologie par défaut ». 13. J. Caune, dans un article très stimulant, a proposé une réflexion sur les rapports entre identité-culture-communication (1991).

14. J'ai proposé dans un texte antérieur de caractériser l'organisation moderne comme ordre symbolique, vu la prégnance du principe de différenciation hiérarchique entre les membres. D'une manière plus générale, las grandes organisations de la société moderne semblent pouvoir être appréhendées comme ordres de communication. Ordres crées «sur le tas » ou ordres préconçus par des architectes spécialisés, les médiations organisationnelles évoquent les traits caractéristiques du langage humain; par l'assemblage de symboles et l'élaboration d'oppositions significatives (préexistantes - clivages sexuels et générationnels - ou créés à l'intérieur de l'organisation - classifications hiérarchiques, distinctions entre services...), elles définissent une facette de l'identité de leurs membres et leur permettent de communiquer, tout en conditionnant par la-même leurs échanges et leurs modes de pensée. (Guigo D, 1942 : 47).

15. L'entreprise, dans un double mouvement, apparaît à la fois comme l'organisation la plus explorée par les chercheurs et comme l'organisation (supposée) la plus apte à répondre aux attentes des chercheurs.

16. Ce qui est dit alors de l'organisation vaut pour la société et réciproquement. Cette perspective est radicalement différente de celles qui envisagent las organisations complexes comme des micro-cultures et dont nous parierons plus loin sous le titre Ethnologie de l'organisation.

17. Il me paraît opportun, ici, de s'interroger avec P. Chaskiel (1991:7) : «Comment analyser le fait que beaucoup de discours "autorisés ». - c'est-à-dire auto-autorisée-se soient centrés sur l'entreprise plutôt que sur la structure d'ensemble, qu'ils soient passés d'un social plutôt « macro » à un social plutôt « micro », au sens où le social serait à percevoir d'abord à travers ses unités, ses organisations-composantes.

18. On peut comprendre cette notion d'intervention-conseil dans un sens proche de celui que donne au concept d'« audit » DE Menonville D., Dubois F, Raffegeau J. L'audit opérationnel. Paris, PUF. 1984, p. 5 :« ensemble de techniques d'information et d'évaluation mises en œuvre au sein d'une démarche cohérente par un professionnel afin de porter un jugement par références à des normes et formuler une opinion sur une procédure ou les modalités de réalisation d'une opération.

19. Il définit la culture comme un "mécanisme de survie basé sur des symboles appris et retransmis socialement. Lesquels comme bien d'autres phénomènes de notre univers possèdent ordre et modèle faisant système » $(1984: 12)$.

20. ou culture d'entreprise " que d'aucuns nomment aussi culte d'entreprise soulignant par lamême la croyance qui s'y attache » (Laurens, 1991 : 94).

21. Dans cet article très éclairant, $P$. Chaskiel parle a ce propos de «rationalisation par l'idéologique ".

22. Comme le montre bien C. Le Moenne (1991:170): «La capacité communicationnelle que sélectionne la culture d'entreprise est ainsi la capacité a adhérer, intérioriser et faire intérioriser, cette culture managériale dont l'axe fondamental est la soumission à la norme et aux valeurs communes, c'est-à-dire la négation des différences, des marginalités, des déviances, des oppositions Elle est stratégie consensuelle en ce qu'elle vise à opérer le consensus sur les valeurs et la culture que véhicule la communication d'entreprise Elle masque les enjeux idéologiques et les Intérêts contradictoires qui caractérisent les cultures « réelles » d'entreprise ».

23. Dans "Vers une gestion culturelle des entreprises", Annales des mines. Gérer et comprendre, septembre 1986, p. 81. 
24. À ce propos, J. Caune -cité également par S. Bonnafous (1992)- proposait de concevoir la culture comme « un « système métabolisant » qui organise et structure les relations existentielles (pratiques et/ou imaginaires), les savoirs, les codes et les modèles de représentation » 1991 : 118). 25. Dans cette optique, je préfère parler de culture de l'organisation (ou de culture de l'entreprise), réservant ainsi le terme culture d'organisation (ou culture d'entreprise) pour les actions de management visant à l'instauration de valeurs-repères et à l'homogénéisation.

26. Par «bloc sociotechnologique ». P. Bouvier entend « un ensemble complexe et opératoire de rapports au travail».

27. Cité par Pascal Dibie, Ethnologie de la chambre àcoucher, Paris, Grasset, 1987.

28. pouvant s'inspirer, entre autres, de méthodes classiques d'analyse de contenu.

29. «Un «topos" est un moyen de la langue pour exprimer une idée socialement partagée et considérée comme une évidence par un groupe particulier (...) Le topos est donc une indication linguistique qui permet de repérer des représentations ou des interprétations sociales de la réalité » (Molitor, 1990 : 22-23).

30. A titre d'exemple, une étude menée dans une organisation sociale avait abouti à une lecture interprétative en termes de statut de participation, de compétence interactive et de processus de figuration, c'est à dire les moyens mis en œuvre par les membres pour éviter à quiconque de perdre la face (voir Goffman). Partant de cela, nous avions pu proposer des extensions prépositionnelles -donc à vocation compréhensive plus large- s'articulant sur les procédures (stratégiques) de fabrication figurative.

31. Goffman. 1984

32. On se reportera aux définitions qu'en donne Erving Goffman.

33. Bien entendu, nous savons qu'il peut y avoir en la matière également diverses opinions, positions et orientations. Ce que je tente, dès lors, de faire c'est de baliser ma propre démarche.

34. On lira avec intérêt Bautier et Nicolas (1991). Dans cet article. Ils traitent de la littérature sur la communication d'entreprise et mettent en évidence un certain nombre de caractères qui peuvent distinguer les experts-conseils des chercheurs. A titre d'exemple, on citera le refus du recours à la théorie, conçue comme un mal nécessaire: «Une des vertus cardinales de ces recours aux dérivés les plus «light» de la science réside dans leur positivité niant les conflits d'interprétation théorique, en les ravalant au rang d'arguties ou en laissant entendre qu'ils les ont résolus, les managers-auteurs tendent à s'imposer alors comme les seuls mécaniciens capables d'affronter la complexité organisationnelle des entreprises avec un outillage que le savoir et l'expérience ont rendu simple et efficace » $(1991: 25)$.

35. On entendra ici par " rôle ", " une fonction particulière qu'il (un individu) peut exercer dans certaines circonstances» (Goffman 1991 :136) -circonstances qui dans notre cas, sont professionnelles. On parlera donc de "rôle » pour désigner "une aptitude ou une fonction » (id. :137)

36. ou travaille à la production de nouvelles normes.

37. Autrement dit, sur le mode métaphorique, qu'il n'y a pas en la matière de spécialités pharmaceutiques ou de recettes de cuisine miraculeuses et intangibles.

38. Risques qui peuvent avoir des retombées sur la pratique ethnosociologique elle-même.

39. Vision qu'il oppose au modèle télégraphique. Pour plus de précisions à ce sujet, voir Winkin Y., La nouvelle communication, Paris, Seuil 1981.

40. «Le point le plus important concernant les clients est qu'à tout instant le consultant (mais cela vaut aussi pour l'ethnosociologue) doit avoir clairement à l'esprit la réponse à la question : «qui est le client?» (E, H Schein. cité par Le Moenne, 1991 :167). En ce qui nous concerne, je vois là une exigence de clarté pour toutes les personnes qui seront «touchées " par la recherche et absolument pas une nécessité de soumission aux idées, souhaits, stratégies du commanditaire.

41. C'est-à-dire les conditions d'exercice de la recherche dans l'organisation. 
42. A ce propos, C. Le Moenne (1991: 168) note judicieusement que le consultant apparaît comme le représentant symbolique de son commanditaire et que, dans le même temps, par le recours à son intervention, ce dernier acquiert une certaine dimension d'extériorité. Cette observation sur une certaine forme d'échange symbolique me semble également valoir pour l'approche ethnosociologique

43. C'est moi qui ajoute.

\section{RÉSUMÉS}

Après avoir rapidement esquissé les fondements et orientations de la démarche ethnosociologique qu'il entend mener, l'auteur envisage la praticabilité de cette dernière dans le champ des organisations et dégage, en les discutant, trois voies majeures d'approche qu'il appelle " ethnosociologie dans l'organisation ", « ethnosociologie d'organisation » et " ethnosociologie de l'organisation ». En les distinguant les unes des autres, il tente de montrer les différences de perspective, de contextes et de paradigmes qui se révèlent constitutives, concrètes, inhérentes à l'investigation ethnosociologique, il engage la réflexion sur le terrain de la pertinence méthodologique et sociale.

After giving a rapide outline of the bases of the ethnosociological approach and the directions which he intends to take, the author considers the praticality of this within (the field of) organizations.

He then distinguishes and discusses three main approaches which the entities "ethnosociology». By making a distinction between them the author tries to show the differences in the perspectives, the contexts and the paradigms which prove to be constituant parts of these very approaches. By examining certain concrete dimensions inherent to ethnosociological investigation in his conclusion, he thereby begins to examine their methodological and social pertinence in the field.

\section{AUTEUR}

\section{GÉRARD DERÈZE}

Gérard Derèze est assistant au Département de communication de l'Université Catholique de Louvain à Louvain-La-Neuve en Belgique. Ses recherches ethnologiques dans le champ de la communication l'amènent à parcourir différents terrains comme les organisations, les milieux sportifs et les médias. Il a co-dirigé récemment le numéro 57 de la revue Réseaux. 